\title{
Design, synthesis and spectral characterization of a new Zinc - Avicularin, a metal flavonol complex and evaluation of its toxicity and antidiabetic efficacy in HFD - Low Dose Streptozotocin Induced Experimental Type 2 Diabetes in Rats
}

\author{
Neeli Parvathi, ${ }^{1}$ Subramanian Iyyampillai, ${ }^{2}$ Sorimuthu Pillai Subramanian ${ }^{1 *}$
}

\section{ABSTRACT}

Objectives: Zinc is an essential trace element with prominent roles in the structural and functional aspects of insulin. Several reports are available on the development of zinc complexes with various ligands to reduce the toxicity of zinc. In the present study, an attempt has been made to synthesize a zinc-avicularin complex and it was subjected to spectral characterization and systematic antidiabetic properties.

Design: The zinc-avicularin complex was synthesized by molar ratio method and characterized by spectral studies such as FT-IR, mass, ${ }^{1} \mathrm{H}$ NMR and ${ }^{13} \mathrm{C}$ NMR. Acute toxicity and dosage fixation studies were carried out as per OCED guidelines. HFD fed -low dose streptozotocin induced experimental type 2 diabetes was chosen as the animal model. The oral glucose tolerance test performed in the control and experimental groups of rats. The levels of fasting blood glucose, hemoglobin, glycosylated hemoglobin ( $\mathrm{HbA1c}$ ), plasma insulin, urine sugar, protein, urea, uric acid and creatinine were determined by established methods. The activities of serum AST, ALT and ALP were assayed.

Results: The spectral studies provide evidence for the complexation between zinc ions with avicularin. Acute toxicity and dosage fixation studies revealed the non toxic nature of the complex and the optimum dose as $5 \mathrm{mg} / \mathrm{kg}$ b.w./rat/day orally for 30 days. The biochemical alterations observed in the experimental diabetic rats were reverted to the physiological range after treatment with zinc-avicularin complex as well as metformin.

Conclusion: The newly synthesized and characterized zinc-avicularin complex is non toxic and elicits significant antihyperglycemic activity which in turn may be due to the insulin stimulatory and/or insulin mimetic activity.

Keywords: Diabetes mellitus, zinc-avicularin complex, spectral characterization, antidiabetic properties.

*Correspondence to:

Dr.S.Subramanian, Professor, Department of Biochemistry, University of Madras, Guindy Campus, Chennai- 600025 subbus2020@yahoo.co.in

Cite This Article: Parvathi, N., lyyampillai, S., Subramanian, S.P. 2020. Design, synthesis and spectral characterization of a new Zinc Avicularin, a metal flavonol complex and evaluation of its toxicity and antidiabetic efficacy in HFD - Low Dose Streptozotocin Induced Experimental Type 2 Diabetes in Rats. Diabesity 6(2): 9-18. DOI: 10.15562/diabesity.2020.64

\section{INTRODUCTION}

Diabetes mellitus (DM) is a multifactorial, multisystemic metabolic disorder that arises due to deficiency (T1DM) and /or efficiency (T2DM) of insulin. It is characterized by chronic elevation in fasting, postprandial, random and glycosylated hemoglobin levels. Chronic hyperglycemia in both T1DM and T2DM is an established risk factor for the development of micro as well as macro vascular complications and premature mortality. Impairment of carbohydrate, fat, protein metabolism and chronic hyperglycemia-induced oxidative stress are the life-threatening consequences of DM. ${ }^{1}$ The currently available drugs for the treatment of DM such as insulin injections and oral antidiabetic drugs having different mechanisms of action often pose undesirable side effects in addition to diminution in response after prolonged use. ${ }^{2}$ This scenario warrants the search for novel drugs capable of controlling chronic hyperglycemia at a low dose and without side effects.
There have been shreds of evidence in the literature to show that the metabolism of several trace elements such as Zinc, Vanadium, Chromium, and Magnesium is altered in DM and these elements might have specific roles in the etiology and progression of diabetes and its secondary complications. ${ }^{3}$ Zinc is an essential trace element succeeding iron in the human body. It is essential for the optimal activity of more than 300 enzymes that are involved in the regulation of vital cellular processes and disturbances of zinc homeostasis have been associated with several diseases including DM. ${ }^{4}$

The discovery of zinc as an essential component of insulin crystals by Scott in 1934, pave a relationship between zinc and diabetes. ${ }^{5}$ The zinc level in pancreatic islets is amongst the highest in the body wherein the concentration is around $20 \mathrm{Mm} .{ }^{6}$ There is a $50 \%$ reduction in pancreatic zinc concentration in diabetic cadavers when compare to non diabetic 
cadavers evidencing a serendipitous association between the zinc, the pancreas and diabetes mellitus. In the pancreatic $\beta$-cells, it is presumed that zinc is essential for the biosynthesis and maturation of insulin. The zinc present in the insulin molecule is coordinated by three nitrogen atoms from histidine and three water molecules in an irregular octahedral environment which is assumed to have a functional structure. In the secretory granules, zinc complexes with insulin combining up to 11.6 ions in a hexamer and this feature implies that zinc both prolongs the duration of action and facilitates the storage of insulin. ${ }^{7}$

Studies using experimental animal models and clinical reports evidenced the notion that zinc deficiency prevails in diabetic conditions and zinc supplementation will benefit diabetes induced zinc status. ${ }^{8}$ For the development of clinically useful metallopharmaceutics, the long-term side effects, clear-cut evidence of target molecule for the in vivo pharmacological action and good pharmacokinetic properties of zinc are vital in the current and future studies. An important advance in the use of zinc compounds as insulin mimics has been the development of various ligands to reduce the toxicity of zinc and also to improve the stability, absorption, utilization and efficiency. ${ }^{9}$

Most of the zinc complexes so far studied for their antidiabetic actions were inadequately absorbed in their inorganic form and require high doses that have been associated with undesirable side effects. To thwart the chronic toxicity and increase gastrointestinal tract absorption of zinc, several complexes have been formulated using ecologically derived non nutrient plant secondary metabolites such as flavonoids as organic ligands and extensively studied for their stability, toxicity and antidiabetic properties. ${ }^{10}$ Flavonoids are broadly classified as flavone, flavonol, flavanone, isoflavone, anthocyanidin and proanthocyanidins. Among these classes, flavonol is known to chelate metal ions due to the presence of multiple hydroxyl groups and $\alpha$-hydroxycarbonyl group. ${ }^{11}$

Avicularin is a bioactive flavonol originally isolated from the leaves of Polygonum aviculare Linn., commonly known as knotweed. ${ }^{12}$ The herb is extensively used in the traditional medicine of all cultures. Avicularin is a quercetin derivative in which the $\alpha$-L-arabinofuranosyl residue is attached at position 3 of quercetin via a glycosidic linkage. Avicularin is non-toxic and reported to possess a wide range of pharmacological properties including antioxidative, anticancer, anti-inflammatory, antiarthritic and hepatoprotective. More recently, it has been reported that avicularin is capable of reversing multidrug resistance in human gastric cancer. ${ }^{13}$ The pharmacokinetic studies evidenced that the oral administration of avicularin is absorbed quickly and the peak plasma concentration reached after approximately $30 \mathrm{~min}$. The concentration of Avicularin remained for a long time (240min). The amount of urinary excretion of Avicularin from 5-36 $\mathrm{h}$ was found to be $77 \%{ }^{14}$

In view of the beneficial and pharmacological properties bestowed with Avicularin, in the present study an attempt has been made to synthesize and characterize a new Zinc-avicularin complex and biochemical evaluate its antidiabetic properties.

\section{MATERIALS AND METHODS}

\section{Chemicals}

Zinc chloride $\left[\mathrm{Zncl}_{2}\right]$, Avicularin (Chemical Formula: $\mathrm{C}_{20} \mathrm{H}_{18} \mathrm{O}_{11}$, Molecular Weight: 434.35) and Streptozotocin were purchased from SigmaAldrich, St. Louis, USA. Ultra-sensitive ELISA kit for rat insulin and C-peptide was purchased from Crystal Chem. Inc. Life Technologies, India. All the other chemicals and reagents used in the present study were of analytical grade.

\section{Analytical instruments}

The FT-IR spectra studies for both free Avicularin and its metal complex were carried out in the solid state as pressed $\mathrm{KBr}$ pellets using a Perkin-Elmer FT-IR spectrophotometer in the range of 400-4000 $\mathrm{cm}^{-1}$. The mass spectrum of the Zinc- Avicularin complex was obtained using Jeol Gc-mate spectrophotometer. The ${ }^{1} \mathrm{HNMR}$ and ${ }^{13} \mathrm{C}$ NMR data of Avicularin and Zinc- Avicularin complex were obtained using Bruker AM-500 instruments at 500.13 and $125.758 \mathrm{MHz}$ respectively. The spectra were recorded without any correction for instrumental characteristics.

\section{Synthesis of macrocyclic Schiff base complexes [ML]}

The zinc-avicularin complex was synthesized as previously reported with slight modifications. ${ }^{15}$ The molar ratio method was followed in the synthesis of the complex (Scheme 1). Because of the very low solubility of these compounds in water, spectrophotometric graded methanol was used. Briefly, a methanolic solution $(25 \mathrm{ml})$ containing hydrated Zinc chloride $(1 \mathrm{mM})$ was gradually added to an equimolar methanolic solution of Avicularin. The $\mathrm{pH}$ of the medium was adjusted to 7.5 with Tris$\mathrm{HCl}$ buffer and the reaction mixture was constantly stirred, refluxed for $8 \mathrm{~h}$ at room temperature. The resulting product was filtered, washed with absolute methanol and dried in vacuum over anhydrous calcium chloride. The golden yellow color solid product obtained was subjected to spectral characterization. 


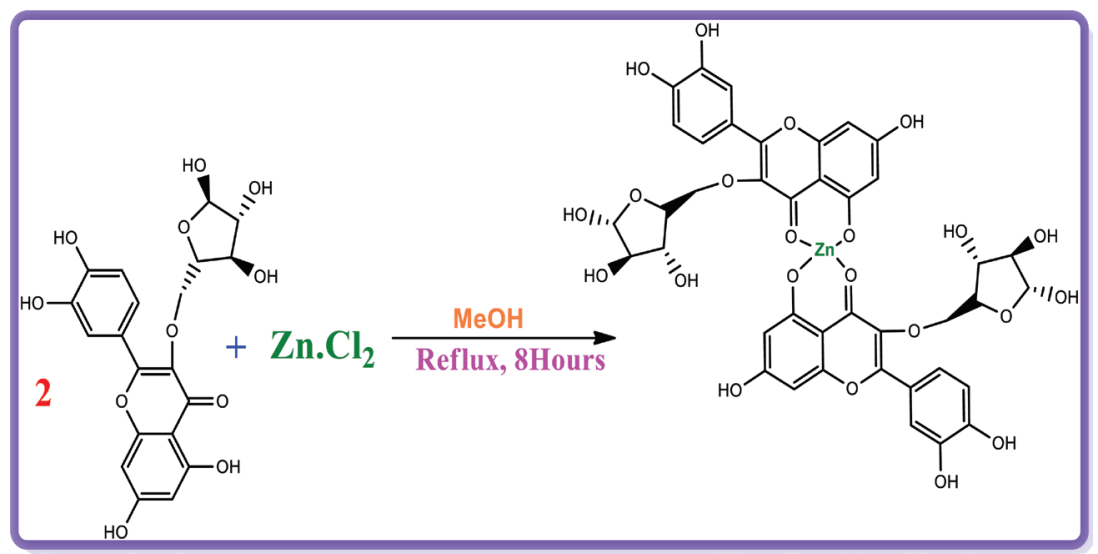

Scheme 1 Schematic representation for the synthesis of Zinc-Avicularin complex

The product percentage of the zinc-avicularin complex was theoretically calculated using the formula:

$\%$ of product $=\left(\frac{\text { Product weight }}{\text { Reactant }}\right) \times$

\section{Experimental animals}

Male albino rats of Wistar strain weighing $(160-180$ g) were procured from Tamilnadu Veterinary and Animal Sciences University (TANUVAS), Chennai. The rats were housed in spacious polypropylene cages lined with husk. The experimental rats were maintained in a controlled environment (12:12 $\pm 1 \mathrm{~h}$ light/dark cycle) and temperature $\left(30^{\circ} \mathrm{C} \pm 2\right)$. Animals were acclimatized to standard husbandry conditions for one week to eliminate the effect of stress before initiation of the experiments. The rats were fed with commercial pellet rats chow (Hindustan Lever Ltd., Bangalore, India), and had free access to water ad libitum.

\section{High-fat diet fed and low-dose STZ-induced} Type 2 diabetes

The High Fat Diet (HFD) was prepared indigenously by using normal pellet diet, raw cholesterol, a mixture of Vanaspati ghee and pure coconut oil (2:1). Briefly, the normal rat pellet diet was powdered by grinding and mixed with $2.5 \%$ cholesterol and a mixture of Vanaspati ghee and coconut oil (5\%). The mixture was made into pellet form and orally fed to rats to induce metabolic syndrome. The rats were divided into two dietary regimens by feeding either normal or HFD for the initial period of 2 weeks. After 2 weeks of dietary management to develop insulin resistance (metabolic syndrome), the groups of rats fed with HFD were intraperitoneally injected with a freshly prepared low dose of STZ (35 mg/kg b.w) dissolved in $0.1 \mathrm{M}$ ice-cold citrate buffer, $\mathrm{pH}$ 4.5. After 3 days of injection with STZ, the experimental rats were screened for fasting blood glucose levels. The experimental rats which show the fasting blood glucose above $250 \mathrm{mg} / \mathrm{dl}$ were considered as diabetic and chosen for further experimental studies. ${ }^{16}$

\section{Acute toxicity and dosage fixation studies}

Acute toxicity studies were performed in normal rats as per OECD guidelines for testing of chemicals. The zinc-avicularin complex in aqueous suspension was administered orally. The rats were observed for four weeks following administration. The change in food consumption, fluid intake, psychomotor activities, body weight gain, changes in the skin, fur, eyes, salivation, diarrhea and lethargy were continuously monitored. Macroscopic examinations were also performed on vital organs. The dosage fixation studies were carried out by administering graded doses of Zinc- Avicularin complex $(2.5,5,10,20$ and $50 \mathrm{mg} / \mathrm{kg}$ b.w./ rat/day) for 30 days to determine the dose-dependent hypoglycemic effect in streptozotocin-induced diabetic rats by monitoring the blood glucose levels periodically.

\section{Experimental protocol}

The animals were divided into four groups namely group 1 Control rats, group 2 diabetes induced rats, group 3 Diabetic rats orally treated with zinc-avicularin complex $(5 \mathrm{mg} / \mathrm{kg}$ b.w./rat/day) as aqueous suspension and group 4 Diabetic rats orally treated with metformin $(200 \mathrm{mg} \mathrm{mg} / \mathrm{kg}$ b.w./ rat/day) in aqueous solution. Each group comprises a minimum of six animals. During the experimental period of 30 days, the food and water intake were monitored periodically along with the change in body weight.

\section{Oral glucose tolerance test}

Overnight fasted rats of all the groups were subjected to oral glucose tolerance test on the penultimate day of sacrifice. Blood samples were collected ( $0 \mathrm{~min})$ from the tail vein of all the groups of rats and $2 \mathrm{~g} / \mathrm{kg}$ b.w./rat, glucose in solution was intragastrically administered. Blood samples were also collected at 30,60,90 and 120 min after the oral glucose load. The blood glucose levels in all the samples were determined by the glucose oxidase method.

\section{Determination of Homeostasis Model Assessment of Insulin Resistance (HOMA-IR)}

As the insulin abnormality cannot be accurately detected by a single determination of insulin levels or from the fasting blood glucose levels, the 
insulin resistance was mathematically derived by HOMA-IR. ${ }^{17}$

$$
\begin{aligned}
\text { HOMA }-\mathrm{IR}= & \frac{\begin{array}{l}
\text { Fasting insulin level }(\mu \mathrm{U} / \mathrm{ml}) \times \\
\text { Fasting blood glucose level }(\mathrm{mg} / \mathrm{dl})
\end{array}}{405}
\end{aligned}
$$

\section{Biochemical analysis}

At the end of 30 days of treatment, rats were fasted overnight, anesthetized, using Ketamine $(80 \mathrm{mg} / \mathrm{kg}$ b.w./rat, i.p.) and sacrificed by cervical decapitation. Blood was collected with and without anticoagulant for plasma and serum separation respectively. Fasting blood glucose, hemoglobin, glycosylated hemoglobin were estimated according to established methods. Ultrasensitive ELISA kit was used for rat insulin and C-peptide. The levels of plasma protein, blood urea, serum creatinine and serum uric acid were also estimated by standardized methods.

\section{Statistical analysis}

All the data were grouped and statistically evaluated with SPSS 11.5 software. Hypothesis testing methods included 'One-way analysis of variance' followed by 'least significant difference' test was used. A value of $p<0.05$ was considered to indicate statistical significance. All results are expressed as mean \pm standard error mean (S.E.M) for six rats in each group.

\section{RESULTS}

The percentage yield for the synthesized zinc-avicularin complex was $72 \%$ and the melting point was (decomp.): $282-285^{\circ} \mathrm{C}$. The elemental analysis carried out to determine the percentage of

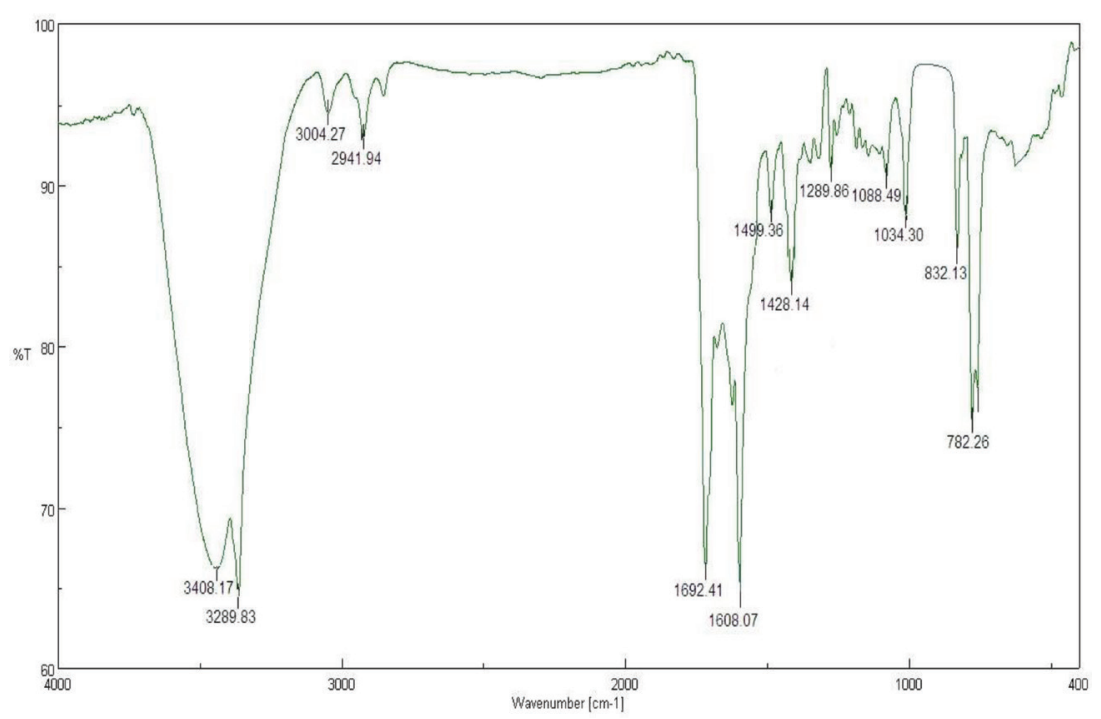

Figure 1 The FT-IR spectrum of free Avicularin
$\mathrm{C}$ and $\mathrm{H}$ present in the synthesized $\mathrm{Zn}(\mathrm{II})$ complex $\left(\mathrm{C}_{40} \mathrm{H}_{34} \mathrm{O}_{22} \mathrm{Zn}\right)$ was analytically calculated and was found to be C, 51.54; H, 3.68; Found: C, 51.59; H, $3.64 ; \%$.

\section{FTIR studies}

The IR spectra of free Avicularin and ZincAvicularin complex is presented as Figure 1 and 2 respectively. The FT-IR spectrum of the free Avicularin ligand showed all the absorption bands correspond to the respective functional groups and some new bands at specific frequencies were observed in the zinc-avicularin complex. The ligand shows intense absorption at $3300-3450 \mathrm{~cm}^{-1}$ corresponds to hydrogen bonding $\mathrm{O}-\mathrm{H}\left(3408 \mathrm{~cm}^{-1}\right)$ in plane stretching vibration. The band observed at $1289 \mathrm{~cm}^{-1}$ is associated with C-O bond. The bands appeared at 1428 and $1499 \mathrm{~cm}^{-1}$ are related to $\mathrm{C}=\mathrm{C}$ vibration of aromatic bonds while the peaks appeared at $3005 \mathrm{~cm}^{-1}$ are related to aromatic C-H bond. A sharp band in the region $462 \mathrm{~cm}^{-1}$ due to the formation of a coordinate bond between the oxygen and the metal ion was observed.

\section{Electron Impact Mass spectral analysis}

The EI mass spectrum of the synthesized zinc complex shows the molecular ion peak at $\mathrm{m} / \mathrm{z}=$ $932[\mathrm{M}]^{+}\left(\mathrm{C}_{40} \mathrm{H}_{34} \mathrm{O}_{22} \mathrm{Zn}\right.$ ) (Figure 3). The peaks at $\mathrm{m} / \mathrm{z}=813,763,695,667,603,581,527,451,419$, $387,347,307,252,178$ and 124 corresponds to the fragments $\mathrm{C}_{36} \mathrm{H}_{28} \mathrm{O}_{18} \mathrm{Zn}, \mathrm{C}_{32} \mathrm{H}_{22} \mathrm{O}_{14} \mathrm{Zn}, \mathrm{C}_{30} \mathrm{H}_{18} \mathrm{O}_{14} \mathrm{Zn}$, $\mathrm{C}_{30} \mathrm{H}_{18} \mathrm{O}_{10} \mathrm{Zn}, \quad \mathrm{C}_{24} \mathrm{H}_{14} \mathrm{O}_{10} \mathrm{Zn}, \quad \mathrm{C}_{18} \mathrm{H}_{10} \mathrm{O}_{10} \mathrm{Zn}$, $\mathrm{C}_{18} \mathrm{H}_{10} \mathrm{O}_{8} \mathrm{Zn}, \quad \mathrm{C}_{18} \mathrm{H}_{10} \mathrm{O}_{6} \mathrm{Zn}, \quad \mathrm{C}_{16} \mathrm{H}_{10} \mathrm{O}_{5} \mathrm{Zn}$ and $\mathrm{C}_{14} \mathrm{H}_{10} \mathrm{O}_{4} \mathrm{Zn}$ respectively.

\section{${ }^{1} \mathrm{H}$ and ${ }^{13} \mathrm{C}$ NMR spectra}

The proton NMR of free ligand and its metal complex recorded using TMS as a reference in the DMSO solvent was presented as Figures 4 and 5 respectively. In the spectrum of the $\mathrm{Zn}$ (II) complex, a sharp singlet appeared at $12.56 \mathrm{ppm}(6 \mathrm{H})$ due to the hydroxyl protons $(-\mathrm{OH})$. A comparison of the chemical shifts of the ligand with that of the complex shows that the signal due to the eight phenolic protons $(\mathrm{OH})$ is reduced to six protons in the complex.

In the ${ }^{13} \mathrm{C}$-NMR spectra of free Avicularin, the presence of the $\mathrm{C}=\mathrm{O}$ characteristic signal at about $170 \mathrm{ppm}$ was observed (Figure 6). A decrease of this signal in the spectrum of the Zinc-avicularin complex along with the appearance of a signal at a lower value (165 ppm) was observed (Figure 7). The free Avicularin exhibited the following peaks which has been assigned as follows 62 (C5"); 75 (C3"); 78 (C2"); 89 (C4"); 93 (C8); 99 (C6); 101 (C10); 106 (C1"); 114 (C5'); 116 (C2'); 119 (C6’); 121 


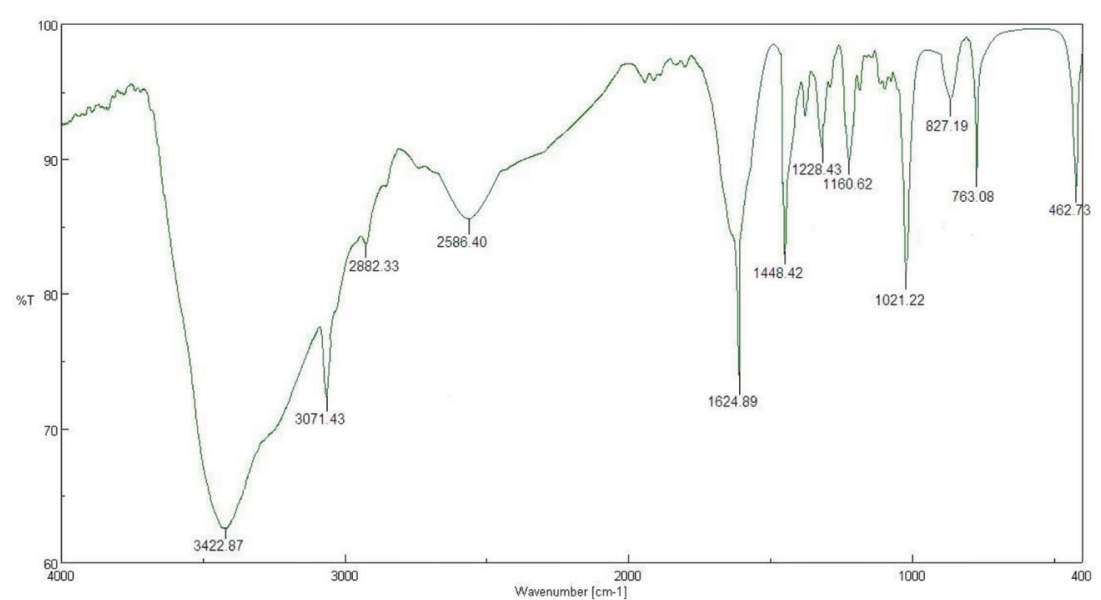

Figure 2 The FT-IR spectrum of Zinc-Avicularin complex

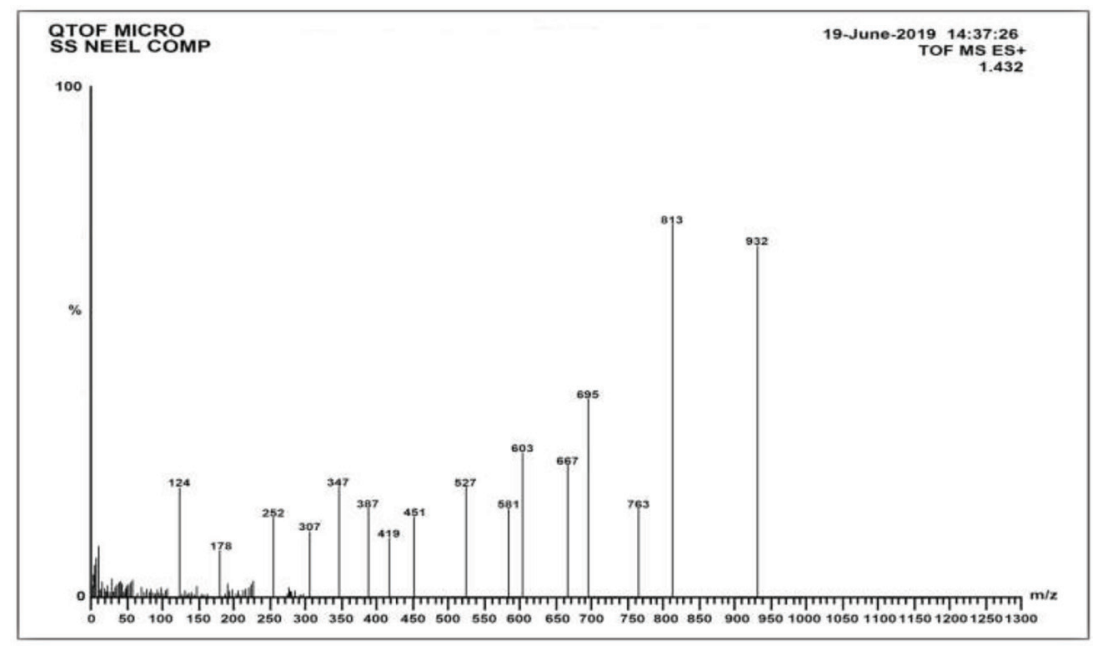

Figure 3 The mass spectrum of Zinc-Avicularin complex
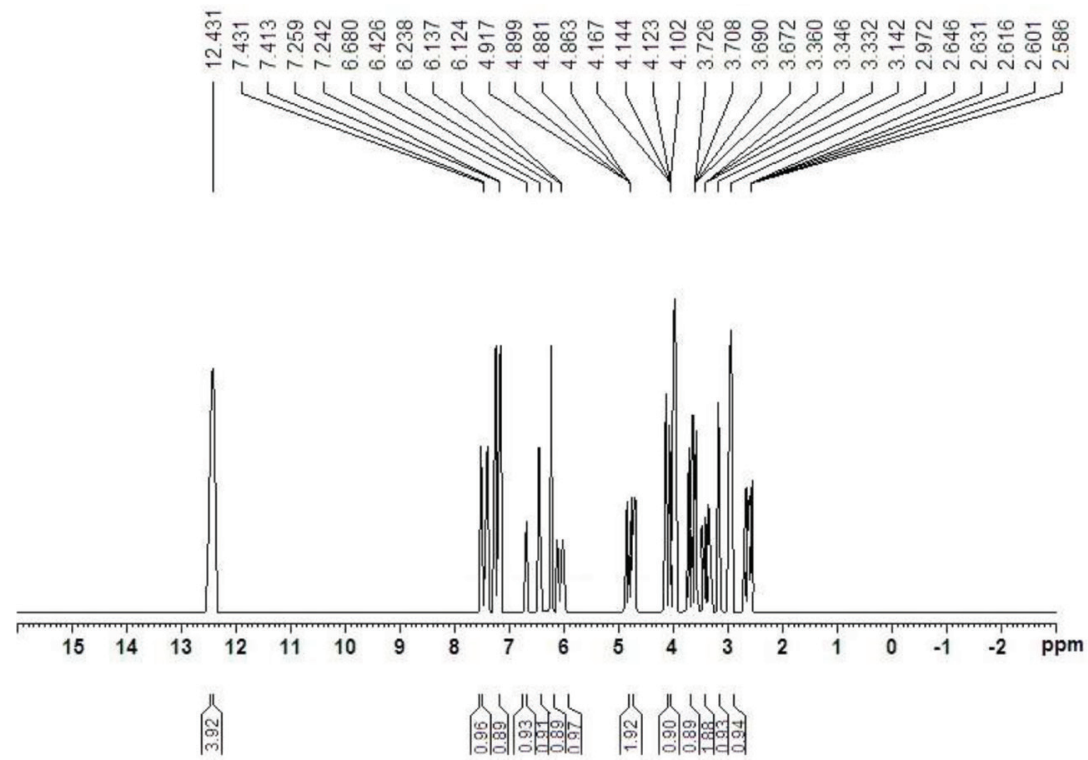

Figure 4 The $1 \mathrm{H}$ NMR of free Avicularin

(C3); 136 (C3'); 144 (C4'); 149 (C2); 153 (C5); 162, $164(\mathrm{C}-\mathrm{OH}) ; 170.0(\mathrm{C}=\mathrm{O})$. However, there were no appreciable changes in all the other signals of this complex while comparing with the parent Avicularin ligand.

The results of the acute toxicity and dosage fixation studies revealed the non toxic nature of the complex and its optimum dose for the treatment of experimental type 2 diabetes in rats as $5 \mathrm{mg} / \mathrm{kg} / \mathrm{bw} /$ rat/day orally for 30 days. The effect of oral administration of the Zn-Avicularin complex as well as metformin on oral glucose tolerance in diabetic rats after an oral glucose load was graphically presented as Figure 8. The blood glucose level before oral glucose load in the diabetic rats was significantly higher than the glucose levels in control rats. In diabetic rats, the blood glucose levels reached a maximum peak at $60 \mathrm{~min}$ and did not return to the basal level even at the end of $120 \mathrm{~min}$. Oral administration with Zn-Avicularin complex to the diabetic group of rats showed a significant decrease in blood glucose concentration at the fasting stage when compared with diabetic rats. After oral glucose load, the blood glucose levels reached a maximum peak at $60 \mathrm{~min}$ but were significantly lower than those in diabetic rats and the peak was reversed to physiological range in the next $60 \mathrm{~min}$ and the efficacy was comparable with metformin treatment.

The HOMA-IR value calculated for the control and experimental groups of rats is presented as Figure 9. HFD-fed low dose STZ induced diabetic rats showed a significant elevation of HOMA-IR and the value was decreased significantly upon treatment with oral administration of Zn-Avicularin complex and the efficacy was comparable with metformin treated diabetic group of rats.

Table 1 portrays the levels of fasting blood glucose, hemoglobin, glycosylated hemoglobin, plasma insulin, C-peptide and the inference for the presence of urine sugar in control and experimental groups of rats. Diabetic rats showed significantly elevated levels of fasting glucose and glycosylated hemoglobin and a concomitant decrease in the levels of hemoglobin and the altered levels were significantly reverted to physiological range in $\mathrm{Zn}$-Avicularin complex treated diabetic rats. Urine sugar detected in the diabetic group of rats was absent in the diabetic group of rats treated with $\mathrm{Zn}$-Avicularin complex as well as metformin.

The levels of plasma protein, blood urea, serum creatinine and uric acid were depicted in Table 2. Diabetic rats showed decreased plasma protein level with concomitantly increased levels of renal function markers namely urea, uric acid and creatinine. The altered levels were significantly reverted to near physiological range in $\mathrm{Zn}$-Avicularin complex and metformin treated diabetic rats. Figure 10 represents the activities of AST, ALT and ALP in 

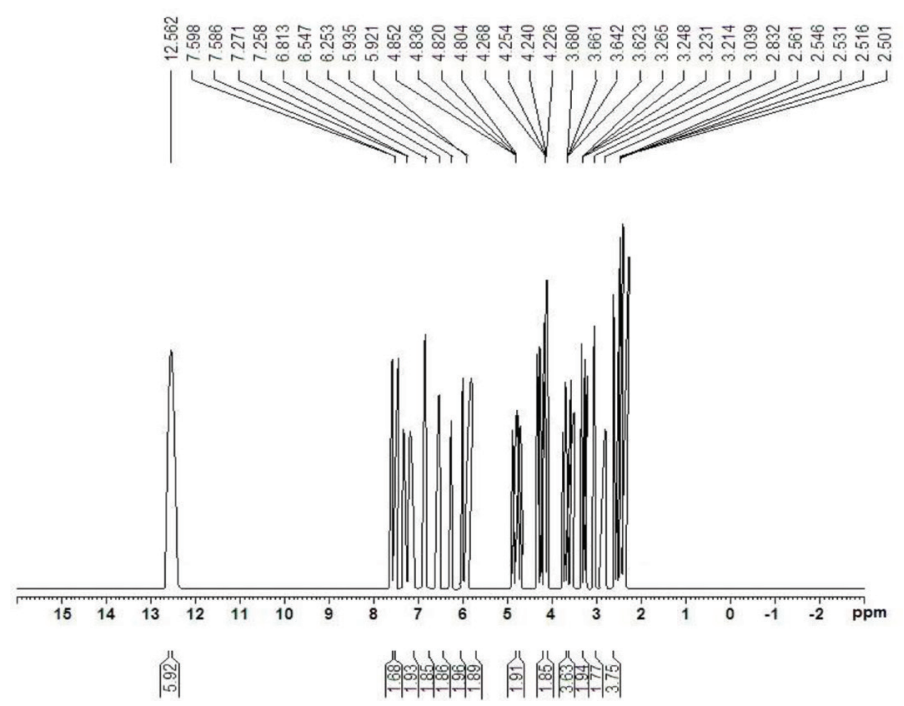

Figure 5 The 1H NMR of Zinc Avicularin complex
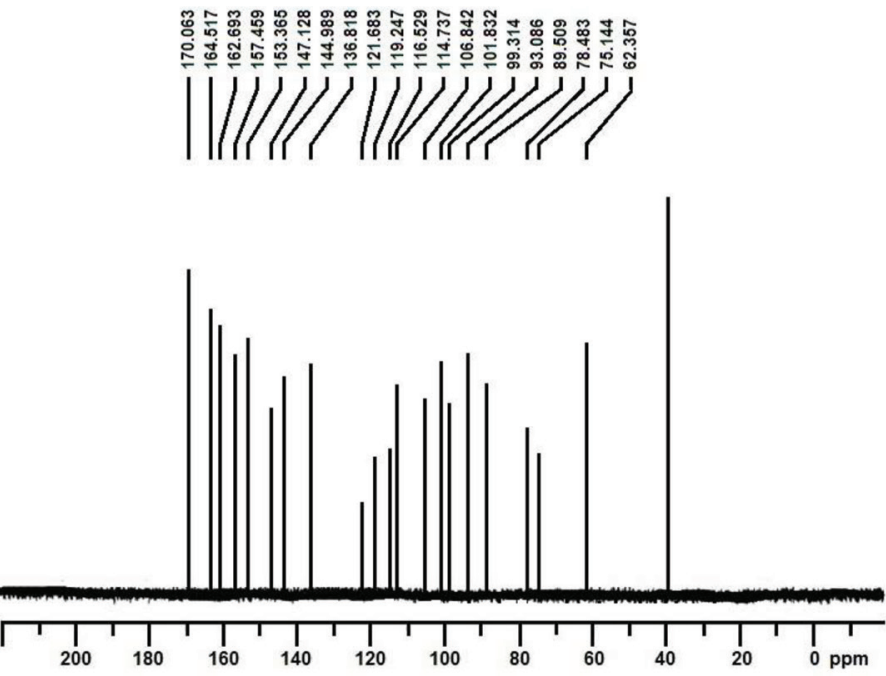

Figure 6 The 13C NMR of free Avicularin

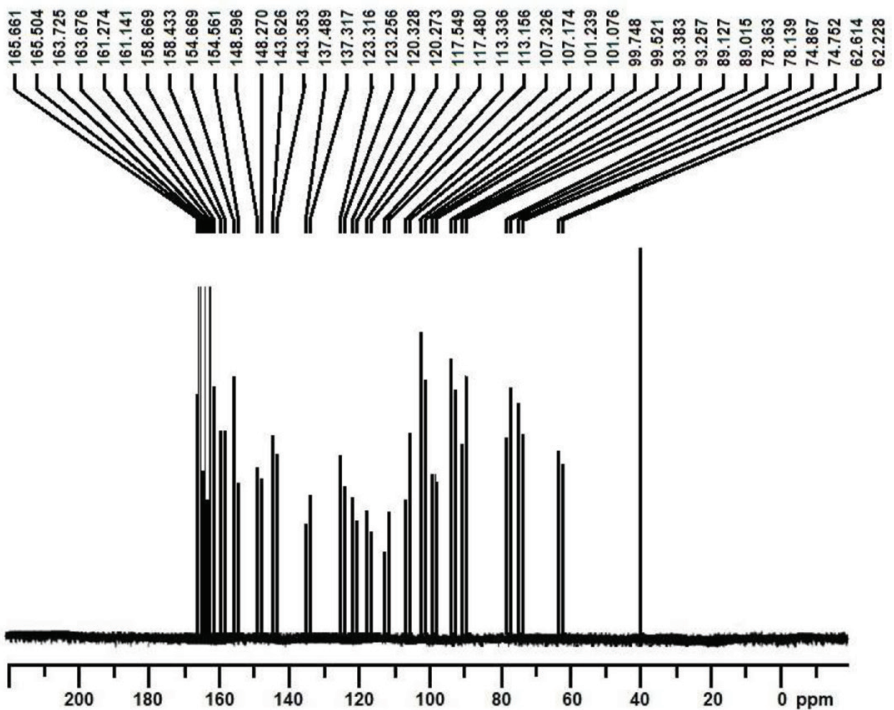

Figure 7 The 13C NMR of Zinc-Avicularin complex

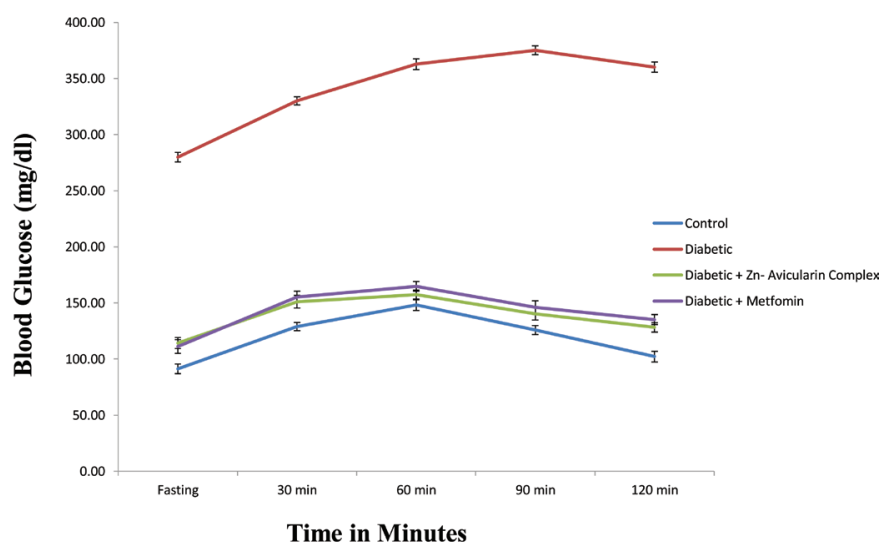

Results are expressed as mean \pm SEM $[n=6]$. One-way ANOVA followed by post hoc test LSD was done. Values are statistically significant at ${ }^{*} p<0.05$. The results were ${ }^{\mathrm{a}}$ compared to control rats and ${ }^{\mathrm{b}}$ compared to diabetic rats.

Figure 8 Effect of Zn- Avicularin complex treatment on the levels of blood glucose after challenging with oral glucose (OGTT)

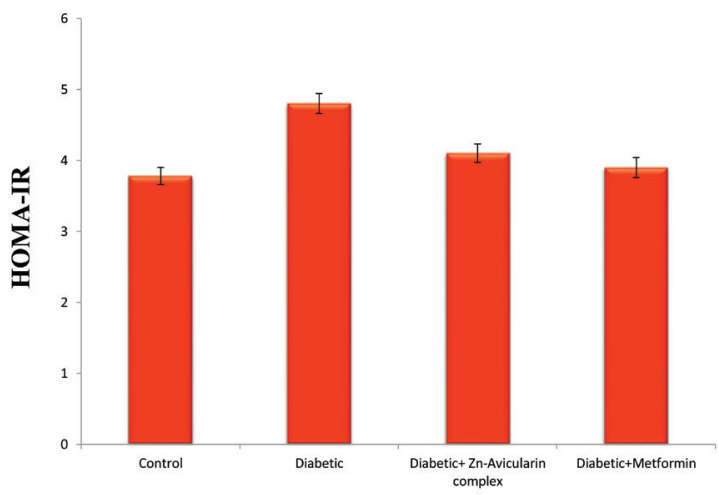

Values are given as mean \pm S.D for groups of six rats in each.

One-way ANOVA followed by post hoc test LSD.

Statistical significance was compared within the groups as follows: ${ }^{\mathrm{a}}$ control rats; ${ }^{\mathrm{b}} \mathrm{diabetic}$ control rats; Values are statistically significant at " $\mathrm{p}<0.05$.

Figure 9 Effect of Zn-Avicularin complex treatment on homeostatic assessment model of insulin resistance (HOMA-IR)

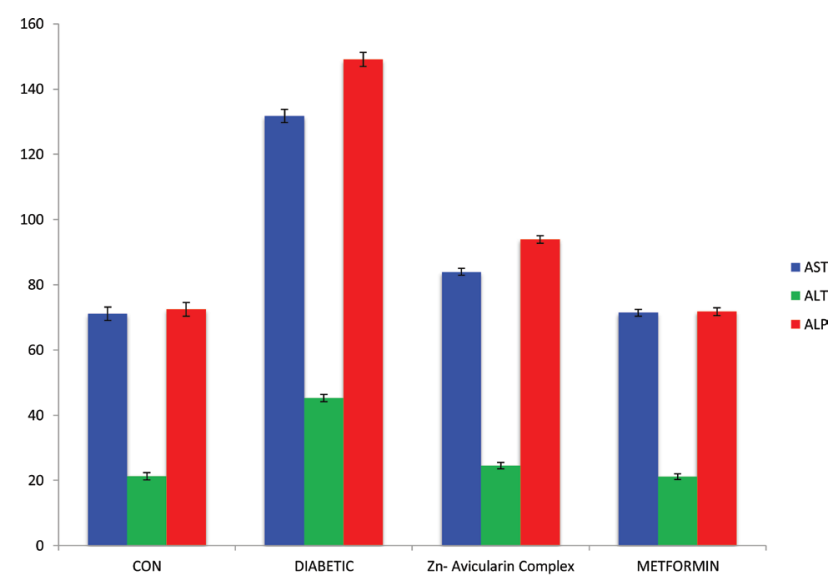

Enzyme activities are expressed as Units: AST and ALT - $\mu$ moles of pyruvate liberated $/ \mathrm{h} / \mathrm{mg}$ protein; ALP - $\mu$ moles of phenol liberated $/ \mathrm{min} / \mathrm{mg}$ protein. Results are expressed as mean $+\mathrm{SEM}[n=6]$. One-way ANOVA followed by post hoc test LSD was done. Values are statistically significant at ${ }^{*} p<0.05$. The results were ${ }^{a}$ compared to control rats and ${ }^{b}$ compared to diabetic rats.

Figure 10 The effect of Zn-Avicularin complex treatment on the activities of AST, ALT and ALP in the serum of experimental groups of rats 
Table 1 Effect of oral treatment of Zn-Avicularin complex on the levels of fasting blood glucose, hemoglobin, glycosylated hemoglobin, plasma insulin and urine sugar in experimental groups of rats after $\mathbf{3 0}$ days of experimental period

\begin{tabular}{|c|c|c|c|c|c|c|}
\hline Groups & $\begin{array}{l}\text { Fasting Blood } \\
\text { Glucose (mg/dl) }\end{array}$ & HbA1c (\% Hb) & $\begin{array}{c}\text { Hemoglobin } \\
(\%)\end{array}$ & $\begin{array}{l}\text { C. Peptide } \\
(\mu \mathrm{U} / \mathrm{ml})\end{array}$ & $\begin{array}{l}\text { Plasma Insulin } \\
(\mu \mathrm{U} / \mathrm{ml})\end{array}$ & Urine sugar \\
\hline Control & $92.21 \pm 5.75$ & $6.01 \pm 0.57$ & $14.51 \pm 0.68$ & $262.81 \pm 1.54$ & $15.37 \pm 1.75$ & Nil \\
\hline Diabetic & $277.25 \pm 16.73^{a^{*}}$ & $10.20 \pm 1.06^{\mathrm{a}^{*}}$ & $9.15 \pm 0.55^{\mathrm{a}^{*}}$ & $125.61 \pm 0.96^{\mathrm{a}}$ & $9.15 \pm 0.44^{\mathrm{a}^{*}}$ & +++ \\
\hline Diabetic + complex & $118.11 \pm 10.13^{b^{*}}$ & $6.42 \pm 0.34^{\mathrm{b}^{*}}$ & $12.25 \pm 0.49^{b^{*}}$ & $210.24 \pm 1.61^{\mathrm{b}^{*}}$ & $12.80 \pm 1.05^{b^{*}}$ & Nil \\
\hline Diabetic + Metformin & $107.05 \pm 7.07^{b^{*}}$ & $6.12 \pm 0.56^{\mathrm{b}^{*}}$ & $12.28 \pm 0.50^{b^{*}}$ & $227.03 \pm 1.45^{\mathrm{b}^{*}}$ & $14.19 \pm 1.28^{b^{*}}$ & Nil \\
\hline
\end{tabular}

Values are given as mean \pm S.D. for six rats in each group. One-way ANOVA followed by post hoc test LSD. Statistical significance was compared within the groups as follows: ${ }^{\mathrm{a}}$ Control rats; ${ }^{\mathrm{b}}$ Diabetic control rats; Values are statistically significant at ${ }^{*} \mathrm{p}<0.05$.

Table 2 Effect of oral treatment of Zn-Avicularin complex on the levels of total protein, blood urea, serum uric acid and serum creatinine in experimental groups of rats

\begin{tabular}{lcccc}
\hline Groups & Total protein $(\mathbf{g} / \mathbf{d l})$ & Blood urea $(\mathbf{m g} / \mathbf{d l})$ & Serum uric acid $(\mathbf{m g} / \mathbf{d l})$ & Serum creatinine $(\mathbf{m g} / \mathbf{d l})$ \\
\hline Control & $8.85 \pm 0.56$ & $22.30 \pm 2.20$ & $2.51 \pm 0.53$ & $0.54 \pm 0.07$ \\
Diabetic & $6.30 \pm 0.39^{\mathrm{a}^{*}}$ & $44.74 \pm 5.16^{\mathrm{a}^{*}}$ & $5.35 \pm 0.67^{\mathrm{a}^{*}}$ & $1.21 \pm 0.31^{\mathrm{a}^{*}}$ \\
Diabetic + complex & $7.22 \pm 0.30^{\mathrm{b}^{*}}$ & $28.32 \pm 1.82^{\mathrm{b}^{*}}$ & $3.09 \pm 0.30^{\mathrm{b}^{*}}$ & $0.67 \pm 0.07^{\mathrm{b}^{*}}$ \\
Diabetic + Metformin & $7.74 \pm 0.58^{\mathrm{b}^{*}}$ & $30.60 \pm 2.93^{\mathrm{b}^{*}}$ & $2.71 \pm 0.22^{\mathrm{b}^{*}}$ & $0.62 \pm 0.08^{\mathrm{b}^{*}}$
\end{tabular}

The results were compared with a Control; biabetic control.Values are given as mean \pm S.D for six rats in each group. One-way ANOVA followed by post hoc test LSD. Values are statistically significant at ${ }^{*} \mathrm{p}<0.05$.

the serum of control and experimental groups of rats. The increased activities of these physiological enzymes were restored to near normal levels in $\mathrm{Zn}$-avicularin complex as well as metformin treated diabetic rats.

\section{DISCUSSION}

The FT-IR spectral data provide valuable information regarding the nature of the functional groups attached to the metal atom. The bonding between the metal and the ligand was confirmed by comparing the IR spectra data of the free Avicularin ligand with the Zinc (II) complex. The data obtained evidence that the modes of absorption and the complexation of the ligand with the metal ion are through hydroxyl hydrogen and carbonyl oxygen. The alteration observed in the sharp absorption band appeared around $3300-3450 \mathrm{~cm}^{-1}$ in the complex, evidenced the involvement of the hydroxyl group in the coordination of the Zinc ion. The formation of a sharp band in the region $462 \mathrm{~cm}^{-1}$ provide further evidence that the binding mode between Zinc and avicularin is through the formation of a coordinate bond between the oxygen and the metal ion. ${ }^{18}$ The EI mass spectral data confirm the formation and molecular structure of the macromolecular Schiff base-Zinc complex.

The co-ordination sites of the ligand have been determined by a careful comparison of the NMR spectra of the complex with that of the parent ligand. The signal due to the eight phenolic protons in the free ligand is reduced to six protons in the complex, suggesting the coordination of the phenolic oxygen to the metal ion after deprotonation. The ${ }^{13} \mathrm{C}$ NMR provides more detailed information about the structure of the ligand and its $\mathrm{Zn}$ (II) complex. The decrease of a signal in the spectrum of the zinc-avicularin complex along with the appearance of a signal at a lower value supported the evidence that the zinc-avicularin complex was formed through carbonyl carbon. Thus, both the ${ }^{1} \mathrm{HNMR}$ and ${ }^{13} \mathrm{C}$ NMR spectroscopic data support the proposed structure of the synthesized zinc-avicularin complex. The NMR analyses obtained were in accordance with a recent report. ${ }^{19}$

Ever since the role of zinc in the structural and its functional aspects of insulin were established, several zinc complexes have been synthesized and studied for their insulinomimetic and insulin sensitizing effects. In order to improve the bioavailability and to reduce the toxicity of zinc, in the present study, Avicularin was used as a ligand to synthesize a new zinc complex. Based on the dose-dependent effect of zinc-avicularin complex on the levels of fasting blood glucose concentration, $5 \mathrm{mg} / \mathrm{kg}$ b.w./rat/day for 30 days was fixed as the optimum dosage for evaluating the antidiabetic properties of the complex in HFD fed-low dose STZ induced experimental type 2 diabetes in rats. 
OGTT is an established measure of effective glucose utilization by the system and it is widely performed to assess the extent of impairment in glucose metabolism. ${ }^{20}$ Due to insufficient insulin secretion coupled with the development of peripheral insulin resistance, the blood glucose concentration before oral glucose load in the diabetic rats was significantly higher than the glucose levels in control rats. However, in the diabetic rats treated with zinc-avicularin complex, the blood glucose levels reached a maximum peak at $60 \mathrm{~min}$ but were significantly lower than those in diabetic rats and the peak was reversed to physiological range in the next 60 min suggesting that the complex possesses the efficacy to maintain glucose homeostasis in hyperglycemic conditions.

The HOMA-IR developed by Matthews et al. $(1985)^{17}$ have been extensively used for the assessment of insulin resistance associated with obesity and appear to be the primary mediator of metabolic syndromes and the development of type 2 diabetes. Experimental diabetic rats showed a significant elevation of HOMA-IR and the value was decreased upon treatment with oral administration of $\mathrm{Zn}$-Avicularin complex and the efficacy was comparable with metformin treated diabetic group of rats. The normalization of HOMA-IR values suggests that the complex improves insulin sensitivity in diabetic rats.

The fasting blood glucose levels are significantly increased in the diabetic group of rats which results from reduced glucose utilization by various tissues, which is a typical condition of insulinopenic. The existence of fasting blood glucose levels within the physiological range observed in the complex treated diabetic rats indicating that the complex treatment has improved the insulin resistance in HFD-STZ induced diabetic rats. The observed increase in the levels of fasting blood glucose ultimately leads to elevated glycosylated hemoglobin levels, because the hemoglobin in circulation binds to glucose during persistently elevated fasting blood glucose levels by an irreversible, non-enzymatic manner to form unusual glycosylated hemoglobin. The glycation of N-terminal valine and numerous lysine residues present in the hemoglobin molecule results in an elevated level of glycosylated hemoglobin with a concomitantly reduced level of hemoglobin in the diabetic group of rats. ${ }^{21}$ The decreased levels of HbAlc along with an improvement in the levels of hemoglobin in the diabetic groups of rats treated with $\mathrm{Zn}$-Avicularin complex evidenced the significant antioxidant properties of $\mathrm{Zn}$-Avicularin complex in addition to its antidiabetic properties.
The observed decrease in the levels of plasma insulin and C-peptide in the diabetic rats were significantly improved to physiological range in the diabetic rats treated with zinc-avicularin complex indicating that the complex treatment may preserve the $\beta$-cell mass and /or cause increased insulin secretion from the functioning pancreatic $\beta$-cells. ${ }^{22}$ Urine sugar which was present in diabetic rats was no longer detected; suggesting that the treatment with zinc-avicularin complex improves glucose homeostasis in diabetic rats and the efficacy of the complex was comparable with diabetic rats treated with metformin.

The observed decrease in the levels of total protein with a concomitant increase in blood urea in the diabetic group of rats may be attributed to increased muscle proteolysis, reduced protein synthesis, the energy dependent processes in the liver tissue and stimulated hepatic gluconeogenesis utilizing the activities of gluconeogenic enzymes. Treatment with the Zn-Avicularin complex significantly controls proteolysis caused by insulin deficiency and improves total protein level to near reference range and this action of the complex was comparable to metformin treatment.

Diabetic rats showed decreased plasma protein levels with concomitantly increased levels of renal function markers such as urea, uric acid and creatinine. The levels were significantly reverted back to near physiological range in $\mathrm{Zn}$-Avicularin complex treated diabetic rats. Decreased protein anabolism with concomitant increased protein catabolism causes remarkable effects in the metabolic functions of renal tissues, which leads to elevated levels of blood urea, serum uric acid and serum creatinine. Protein catabolic end products namely urea, creatine and phosphocreatine breakdown product creatinine, which are considered as energy storage compounds in muscle tissues. The elevated levels of blood urea, serum uric acid and serum creatinine suggest the impairment in the renal function to excrete urea, uric acid and creatinine. These renal markers were significantly reduced in $\mathrm{Zn}$-Avicularin complex treated diabetic rats, indicating the beneficial effect of the complex in ameliorating diabetes associated renal secondary complications which otherwise may lead to diabetic nephropathy. ${ }^{23}$

In addition, the serum creatinine levels also depend on the ability of the renal tissues to excrete creatinine and hence its level is often used as a variable not only to assess impairment of renal function but also as a clinical endpoint to detect treatment related toxic effects of drugs on renal functions. It has been reported that the increased proteolysis during chronic hyperglycemia occurs 
as a result of deranged glucagon-mediated regulation of cAMP formation in insulin deficiency. The observed decrease in the levels of serum creatinine in Zn-Avicularin complex treated experimental diabetic groups of rats suggests the proper regulation of carbohydrate and protein metabolism. ${ }^{24}$ Additionally, the normalized serum creatinine levels indicate the renoprotective nature of the Zn-Avicularin complex.

The cytosolic enzymes such as AST, ALT and membrane bound ALP are the established physiological markers normally present in low levels in serum and their activities elevated significantly during tissue damage. A significant rise in ALT activity indicates the hepatocellular damage due to the diabetogen and is usually accompanied by a concomitant rise in AST activity. ${ }^{25}$ The observed increase in the activity of these enzymes in the serum of diabetic rats may be due to the leakage of these enzymes from the cytosol into the bloodstream as a consequence of tissue damage. The reversal of AST, ALT and ALP activities in zinc-avicularin complex treated diabetic rats towards near normalcy indicates the tissue protective nature of the complex.

\section{CONCLUSION}

The present study portraits the synthesis of a new Zinc - Avicularin complex and its structural characterization by spectral studies. The acute toxicity and dosage fixation studies revealed the non toxic nature of the synthesized complex and its optimum dose for the treatment of experimental type 2 diabetes in rats. The results of the vital biochemical parameters evidenced the insulin stimulatory as well as insulin- mimetic properties of the Zinc Avicularin complex. Further detailed studies are in progress to understand the molecular mechanisms involved in the process of maintaining normoglycemia in diabetic conditions.

\section{CONFLICT OF INTEREST}

The authors declare that there is no conflict of interest.

\section{REFERENCES}

1. Hao W, Lu C, Judy B, de H, and Robertina G. Targeting Oxidative Stress in Diabetic Complications: New Insights. J. Diabetes Res.2018;1-2.

2. Arun C, Chitharanjan D, Vijaya SRD, Shashank K, Aditya C, Rahul R, Asween M, Nawal SS, Maria TM, Kevin K, Appalanaidu S, Alexandria B, Naveen P, Chaitanya K, Musham, Govinda PL, Wasique M. Clinical Review of Antidiabetic Drugs: Implications for Type 2 Diabetes Mellitus Management. Front Endocrinol (Lausanne). 2017; 8(6):1-8.
3. Namrata S, Jeanne FGS, Natasha B, Prageet K, Sachdev. Trace element status in type 2 diabetes: A meta-analysis. J Clin Diagn Res. 2018; 12(5): OE01-OE08.

4. Nazanin R, Richard H, Roya K, Rainer S. Zinc and its importance for human health: An integrative review. J Res Med Sci. 2013; 18(2): 144-157.

5. Scott DA. Crystalline insulin. Biochem J. 1934; 28: 1592-1602.

6. Mariea DB, Daisy MM, Chris JD, Carol JL, Peter DZ, Toby Coates. Zinc and Zinc Transporter Regulation in Pancreatic Islets and the Potential Role of Zinc in Islet Transplantation. Rev Diabet Stud. 2010; 7(4): 263-274.

7. Emdin, SO, Dodson GG, Cutfield JM, Cutfield SM. Role of zinc in insulin biosynthesis. Some possible zinc-insulin interactions in the pancreatic B-cell. Diabetologia. 1980; 19:174-182.

8. Jansen J, Karges W, Rink L. Zinc and diabetes-clinical links and molecular mechanisms. J. Nutr. Biochem. 2009; 20: 399-417.

9. Priyanga R, Shehani P, Priyadarshani G, Prasad K, Godwin R. Constantine. Zinc and diabetes mellitus: understanding molecular mechanisms and clinical implications. Daru. 2015; 23(1): 1-13.

10. Gopalakrishnan V, Iyyam Pillai S, and Subramanian SP. Synthesis, Spectral Characterization, and Biochemical Evaluation of Antidiabetic Properties of a New ZincDiosmin Complex Studied in High Fat Diet Fed-Low Dose Streptozotocin Induced Experimental Type 2 Diabetes in Rats. Hindawi Publishing Corporation Biochemistry Research International.2015; 11:1-11.

11. Gregory M, Karthik S, Qaisar M, Rajendran KS, Dariusz K, Piotr K, Gregory F. Secondary Metabolites in the Green Synthesis of Metallic Nanoparticles. Materials (Basel) 2018;11(6): 1-25.

12. HOU KZ. The dictionary of genera of seed plants Chinese. Beijing: Science Press, 1982: 291.

13. Guo XF, Liu JP, Ma SQ, Zhang P and Sun WD: Avicularin reversed multidrug-resistance in human gastric cancer through enhancing Bax and BOK expressions. Biomed Pharmacother. 2018;103:67-74.

14. Gabriela AB, Dayana RG, Sherwin KB Sy, Alexander V, Ravi SPS, Denise BS, Elza K, Hartmut D, Norberto PL, Andrea D. Pharmacokinetic Evaluation of Avicularin Using a Model-Based Development Approach. Planta Med 2015; 81: 373-381.

15. Christine L, Laetitia D, and Jean-PC. Spectroscopic and Theoretical Studies of the $\mathrm{Zn}$ (II) Chelation with Hydroxyflavones. J. Phys. Chem. A 2006;110: 12494-12500.

16. Nath S, Ghosh SK, \& Choudhury Y. A murine model of type 2 diabetes mellitus developed using a combination of high fat diet and multiple low doses of streptozotocin treatment mimics the metabolic characteristics of type 2 diabetes mellitus in humans. J Pharmacol Toxicol Methods. 2017; 84:1-30.

17. Matthews DR, Hosker JP, Rudenski AS, et al. Homeostasis model assessment: Insulin resistance and beta-cell function fromfasting plasma glucose and insulin concentrations in man. Diabetologia. 1985; 28:412-19.

18. McAuliffe CA, Parish RV, Ashmawy FM, Issa RM and Amer SA. Synthesis and Characterisation of some New Manganese(i1) and Manganese( 111) Complexes of Tetradentate Schiff-base Ligands and their Reaction with Molecular Oxygen. J. Chem. Soc. Dalton Trans. 1987;2009- 2012.

19. Zhu X, Qiu Z, Ouyang W, Miao J, Xiong P, Mao D, Feng K, Li M, Luo M, Xiao H, Cao Y. Hepatic transcriptome and proteome analyses provide new insights into the regulator mechanism of dietary avicularin in diabetic mice. Food Res Int. 2019;125:108570: 1-11.

20. Katrin H, Richard M, Watanabe, Darko S, Richard NB. OGTT-derived measures of insulin sensitivity are confounded by factors other than insulin sensitivity itself. Obesity. 2008; 16(8): 1938-1945. 
21. Nathan DM, Turgeon H, and Regan S. Relationship between glycated haemoglobin levels and mean glucose levels over time. Diabetologia. 2007;50: 2239-2244.

22. Wajchenberg BL. $\beta$-Cell Failure in Diabetes and Preservation by Clinical Treatment. Endocrine Reviews. 2007;28(2):187-218.

23. Ju Sung L, Ah Young L, Norman G. Quilantang, Paul JLG, Eun JC, Sanghyun Lee. Anti-oxidant activity of avicularin and isovitexin from Lespedeza cuneata. J Appl Biol Chem. 2019; 62(2): 143-147.

24. Fujimori K, \& Shibano M. Avicularin, a plant flavonoid, suppresses lipid accumulation through repression of $\mathrm{C} /$ EBPa-activated GLUT4-mediated glucose uptake in 3T3L1 cells. Journal of Agricultural and Food Chemistry. 2013; 61(21):5139-5147.
25. Rao GM, Morghom LO, Kabur MN, Mohmud BM, and Ashibani K. Serum glutamic oxaloacetic transaminase (GOT) and glutamic pyruvic transaminase (GPT) levels in diabetes mellitus. Indian J. Med Sci.1989;43(5), 118-21.

\section{cc) (1) $(9)$ $\mathrm{Br} \mathrm{NC} \mathrm{NO}$}

This work is licensed under a Creative Commons Attribution-Non Commercial-No Derivatives 4.0 International License. To view a copy of this license, visit http://creativecommons.org/licenses/by-nc-nd/4.0/ 